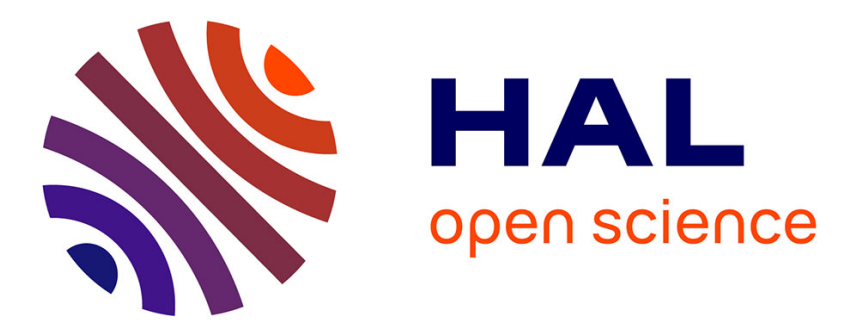

\title{
Performance Evaluation of MIMO-based MAC/PHY cross-layer design in multi-hop ad hoc networks
}

\author{
Hakim Badis, Abderrezak Rachedi
}

\section{To cite this version:}

Hakim Badis, Abderrezak Rachedi. Performance Evaluation of MIMO-based MAC/PHY cross-layer design in multi-hop ad hoc networks. IEEE 11th International Conference on Wireless and Mobile Computing, Networking and Communications (WiMob), Oct 2015, Abu-Dhabi, United Arab Emirates. hal-01201778

\section{HAL Id: hal-01201778 \\ https://hal.science/hal-01201778}

Submitted on 14 Oct 2015

HAL is a multi-disciplinary open access archive for the deposit and dissemination of scientific research documents, whether they are published or not. The documents may come from teaching and research institutions in France or abroad, or from public or private research centers.
L'archive ouverte pluridisciplinaire HAL, est destinée au dépôt et à la diffusion de documents scientifiques de niveau recherche, publiés ou non, émanant des établissements d'enseignement et de recherche français ou étrangers, des laboratoires publics ou privés. 


\title{
Performance evaluation of MIMO-based MAC/PHY cross-layer design in multi-hop ad hoc networks
}

\author{
Hakim Badis and Abderrezak Rachedi \\ University Paris-Est Marne-la-Vallée (UPEM) \\ Gaspard Monge Computer Science Laboratory (UMR 8049 LIGM) \\ Champs sur Marne, France \\ Email: \{badis, rachedi\}@u-pem.fr
}

\begin{abstract}
In Single-Input Single-Output (SISO) wireless multi-hop networks, when two or more stations attempt to transmit at the same time in the same vicinity area, a collision will occur. In this case, the collided packets are discarded and then retransmitted later. In order to deal with collision and to maximize the system's throughput, we propose a new cross-layer design based on multiple transmit and receive antennas (MIMO) capabilities, called MIMOMAX. Transmit filter, receive filter and channel state information (CSI) are exchanged between the PHY and MAC layers to optimize the scheduling of simultaneous transmissions on the same channel. The performance evaluation is conducted using OPNET simulation tool. The results obtained through extensive simulations are compared with those of IEEE $802.11 \mathrm{~b} / \mathrm{g} / \mathrm{n}$ single-hop and multi-hop wireless networks. We show that MIMOMAX is less efficient (low throughput and long delays) for single-hop networks. This is due to an underutilization of channel Degrees of Freedom (DoFs). However, it always performs better (high throughput and low packet delay) than the $802.11 \mathrm{~b} / \mathrm{g} / \mathrm{n}$ wireless multi-hop networks: up to $30 \%$ and $87 \%$ as improvements in network throughput compared to the IEEE 802.11n and IEEE 802.11g networks respectively. In addition, we illustrate that MIMOAX is robust and resists to the early network saturation problem.
\end{abstract}

Keywords-Multi-hop ad hoc network, Cross-layer, MIMO, Performance analysis.

\section{INTRODUCTION}

IEEE 802.11n [1] is the first wirless local area network (WLAN) standard based on MIMO-OFDM systems. It significantly improves range, data rate (up to $600 \mathrm{Mbit} / \mathrm{s}$ ) and link reliability than the current WLAN amendments $(802.11 \mathrm{a} / \mathrm{b} / \mathrm{g})$. Such advantages can be used to enhance the quality of service (QoS) of the high-data-rate applications in multihop radio networks. Indeed, many studies [2], [3], [4], [5] have investigated the impact of IEEE 802.11n MAC and Physical protocols on the performance of multi-hop wireless networks. It was noticed a significant improvement in terms of throughput. However, the IEEE 802.11n MAC protocol does not fully exploit the capabilities of the Physical layer (PHY) like multiple simultaneous transmissions from multiple nodes in the same collision area. Thus, some recent works [6], [7], [8], [9], [10], [11], [12], [13] on cross-layer MAC protocols design have been proposed to offer the functionality of parallel transmissions without interference. The idea is to distribute the spatial degrees of freedom (DoFs) between spatial multiplexing combined with beamforming and spatial nulling in the presence of concurrent transmissions. However, very little simulation results in those works have been done to illustrate the positive impact of MIMO systems on network performance. This paper presents MIMOAX, a new cross-layer design based on MIMO systems to facilitate the PHY-MAC dialogue. The cross-layer parameters include: transmit filters, receive filters and channel state informations (CSI). MIMMAX performance evaluation is conducted through simulations using the simulator OPNET [14]. We carry out a comparative study with IEEE $802.11 \mathrm{~b} / \mathrm{g} / \mathrm{n}$ single-hop and multi-hop wireless networks in terms of overall throughput and end-to-end delay.

\section{A. Contributions}

The main contributions of this paper can be summarized as follows:

- We propose a new cross-layer framework for MIMO ad hoc networks combining spatial-division multiplexing, beamforming and spatial nulling;

- We develop and integrate a new module for OPNET simulator which implement our cross-layer functioning. The module is available for downloading at [15];

\section{B. Outline}

Section II describes the proposed cross-layer framework, MIMOMAX. The simulation model and performance results are given in Section III. The final section concludes this paper with remarks for future work.

\section{MIMOMAX: MIMO-BASED MAC/PHY CROSS-LAYER DESIGN}

In this section we briefly describe our MAC centric crosslayer approach. We assume the following hypotheses:

- $\quad$ Each node is equipped with $M$ antennas;

- The MIMO channel matrix $H_{M \times M}$ can be estimated using training symbols integrated in control packet headers;

- The MIMO channel matrix $H_{M \times M}$ remains static during the 4-way handshake RTS/CTS/DATA/ACK;

- The MIMO channel matrix $H_{M \times M}$ is identical in uplink and downlink directions, i.e., $H_{M \times M}$ is reciprocal.Otherwise, a calibration process is required [16].

In order to achieve higher overall network throughput using multiple antennas, our scheme applies spatial-division 
multiplexing with beamforming techniques (B-SDM) at the PHY layer. B-SDM [17] consists on transmitting multiple spatially orthogonal data streams by TX beamforming, while the receiver uses the zero-forcing (ZF) [17] to cancel interference using some of its spatial receive degrees of freedom. This transmission technique improves the received signal strength and maximizes the MIMO channel capacity. For this purpose, the MIMO channel $H$ between the TX and RX antennas is orthogonalized by using singular value decomposition (SVD) [18]. The SVD decomposition can be expressed as

$$
H=U \Lambda V^{*},
$$

where

$$
\begin{aligned}
& U=\left[U_{1} U_{2} \cdots U_{M}\right], \\
& V=\left[V_{1} V_{2} \cdots V_{M}\right], \\
& \Lambda=\operatorname{diag}\left(\lambda_{1}, \lambda_{2}, \cdots, \lambda_{M}\right) \quad\left(\lambda_{1} \geq \cdots \geq \lambda_{M}\right) .
\end{aligned}
$$

Here, diag $(\cdot)$ denotes a diagonal matrix of real and nonnegative singular values. Each singular value, $\lambda_{i}$, represents the received signal strength (RSS) of the transmitted data stream at the receiver. $U$ and $V$ are $M \times M$ orthonormal matrices. The $M$ columns of $U$ and $V$ are the left-singular and right-singular vectors of $H$, respectively. To form an orthogonal multi-beam space between the TX and RX antennas, we use the right-singular vectors $(V)$ as TX filter. At the receiver side, we simply multiply the received signal by the conjugate transpose of left-singular vectors $\left(V^{*}\right)$. To ensure a good understanding, let us denote: the vector of transmitted streams by $s(t)=\left[s_{1}(t) s_{2}(t) \cdots s_{k}(t)\right]^{T}(k \leq M)$, the vector of received streams before filtering by $r(t)=$ $\left[r_{1}(t) r_{2}(t) \cdots r_{k}(t)\right]^{T}$, the vector of received streams after filtering by $w(t)=\left[w_{1}(t) w_{2}(t) \cdots w_{k}(t)\right]^{T}$, and the noise vector by $n(t)$. The signal $s(t)$ transmitted by the source is first multiplied by the TX filter $(V)$, and then by the channel matrix $H$. The received signal before filtering can be written as:

$$
\begin{aligned}
r(t) & =H V s(t)+n(t) \\
& =U \Lambda V^{*} V s(t)+n(t) \\
& =U \Lambda s(t)+n(t) .
\end{aligned}
$$

By applying the RX filter $U^{*}$ at the receiver, we obtain

$$
\begin{aligned}
w(t) & =U^{*} r(t) \\
& =U^{*} U \Lambda s(t)+U^{*} n(t) \\
& =\Lambda s(t)+U^{*} n(t) .
\end{aligned}
$$

As a result of $\lambda_{1} \geq \lambda_{2} \geq \cdots \geq \lambda_{M}$, we have $\operatorname{RSS}\left(w_{1}(t)\right) \geq \operatorname{RSS}\left(w_{2}(t)\right) \geq \cdots \geq \operatorname{RSS}\left(w_{k}(t)\right)$. The concept of B-SDM is illustrated in Figure 1.

The maximum number of spatial streams supported on a MIMO channel is $M$ (remember that each node is equipped with $M$ antennas). When a source node has less spatial streams to transmit, other source nodes sharing the same channel can simultaneously transmit in the limit of the available degrees of freedom (DoFs) of the channel. Thus, new transmissions do not interfere with the transmissions in progress. To do this, each potential source node must ensure that its signal will be canceled by all active receiving nodes in its collision area, and

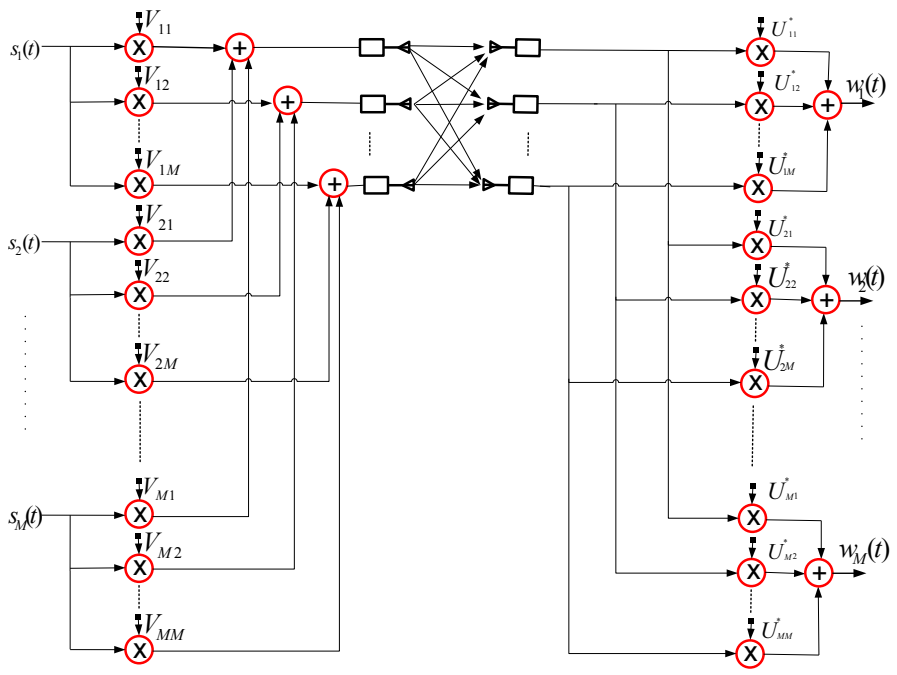

Fig. 1. Spatial-division multiplexing with beamforming technique.

each potential receiving node must ensure that it is able to cancel the signals of all active source nodes in its collision area. This problem can be formulated as an optimization problem and resolved at the MAC layer based on the crosslayer parameters transmitted by the PHY layer.

The physical (PHY) and MAC layers collaborate to exchange cross-layer parameters: TX/RX filters and the channel state information (CSI). Each node should maintain a list of the current transmitting and receiving neighbors, their TX/RX filters and CSIs. RTS/CTS/DATA/ACK frames exchange could be used for this purpose. Additional MAC header fields are added for knowledge discovery process. Each TX/RX filter in the list has a validity time equal to the time specified in the corresponding MAC header duration field.

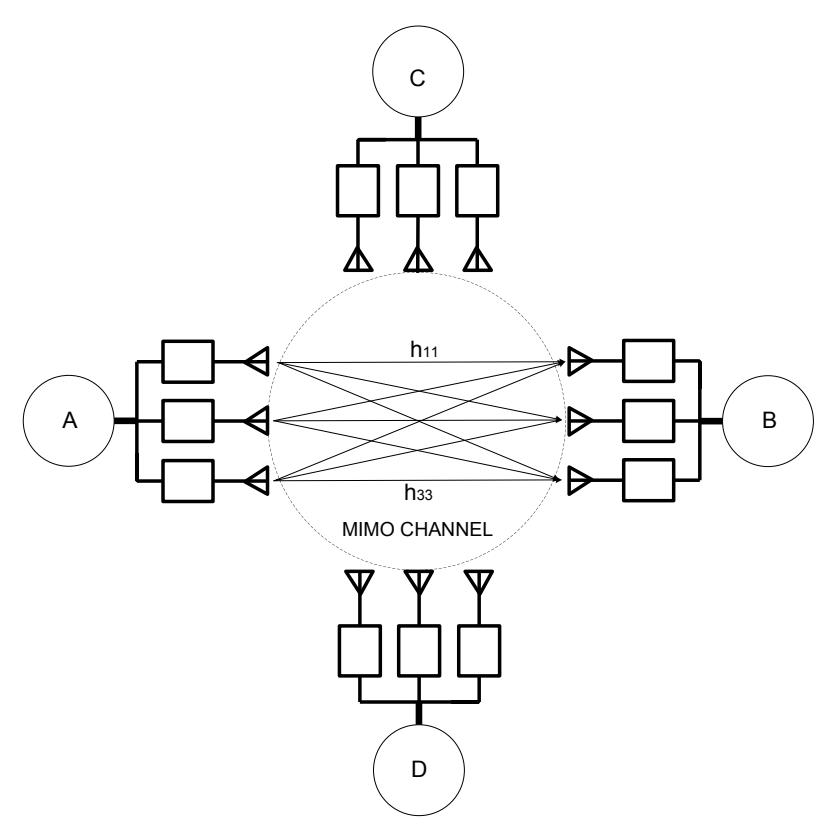

Fig. 2. wireless network scenario. 
To illustrate our protocol functioning, let us consider the network shown in Figure 2. Each node is equipped with 3 antennas, and therefore the number of DoFs available in the MIMO channel is limited to 3 . We suppose that a node A wants to communicate with $\mathrm{B}$ and $\mathrm{C}$ wants to communicate with $\mathrm{D}$. First of all, the node A sends a Request-to-Send frame (RTS)

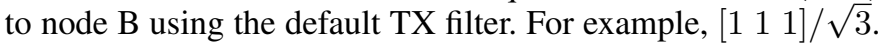
The vector is normalized to have the same average power gain over all transmitting antennas. When the node $\mathrm{B}$ receives the RTS frame, it estimates the MIMO channel matrix between the nodes $\mathrm{A}$ and $\mathrm{B}, H_{A B}$. Using the SVD decomposition, and as explained above: the RX filter is set to the first column of $U_{B, 1}^{*}$ and the TX filter to $V_{B, 1}$. Next, The node B sends a Clear-toSend frame (CTS) to node A using its TX filter $V_{B, 1}$. When the node A receives the CTS frame, it estimates the channel matrix $H_{B A}$ and extracts its channel matrix $H_{A B}$ from the MAC header. To ensure the channel reciprocity, a calibration process is required [16]. Using the SVD decomposition, the node B sets its RX filter to $U_{A, 1}^{*}=U_{B, 1}^{*}$ (because $H_{A B}=H_{B A}$ ), and the TX filter to $V_{A, 1}$. When the nodes $\mathrm{C}$ and D hear the RTS/CTS frames, they estimate the effective channels $\left\{H_{A C}, H_{B C}\right\}$ and $\left\{H_{A D}, H_{B D}\right\}$, respectively. These values should be taken into account in the adjustment of the TX/RX filters for the duration of time specified in the MAC header duration field. Finally, the node $\mathrm{A}$ sends a data frame using its TX filter $V_{A, 1}$. After successful reception, node $B$ replies with an ACK using its TX filter $V_{B, 1}$. Figure 3 shows the above steps. Note that the node A can transmit three simultaneous data streams.

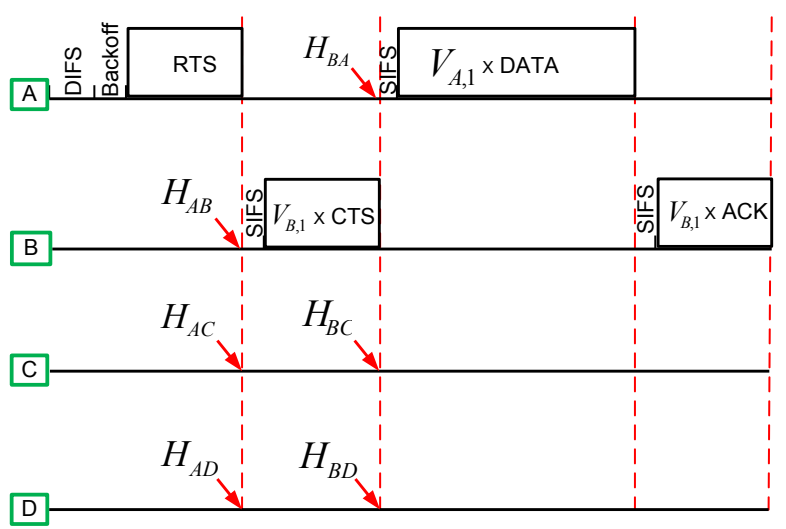

Fig. 3. Four-way handshake to establish communication when the channel is idle.

If the node $\mathrm{C}$ wants to initiate a second transmission to the node $\mathrm{D}$ without interrupting the active session between the nodes $\mathrm{A}$ and $\mathrm{B}$, it should adjust its TX filter $V_{C, 1}$ such that its transmitted signal will be nullified at $\mathrm{A}$ and $\mathrm{B}$. In other words, the two constraints should be satisfied by $V_{C, 1}$ are

$$
\left\{\begin{array}{l}
U_{A, 1}^{*} H_{C A} V_{C, 1}=0 \\
U_{B, 1}^{*} H_{C B} V_{C, 1}=0
\end{array}\right.
$$

The node $\mathrm{D}$ adjusts its weight vector $V_{D, 1}$ in the same way that the node $\mathrm{C}$. Figure 4 illustrates the communication process between $\mathrm{C}$ and $\mathrm{D}$.

Utilizing MIMO with MIMOMAX in wireless ad hoc networks is similar to using directional antennas. Both are able

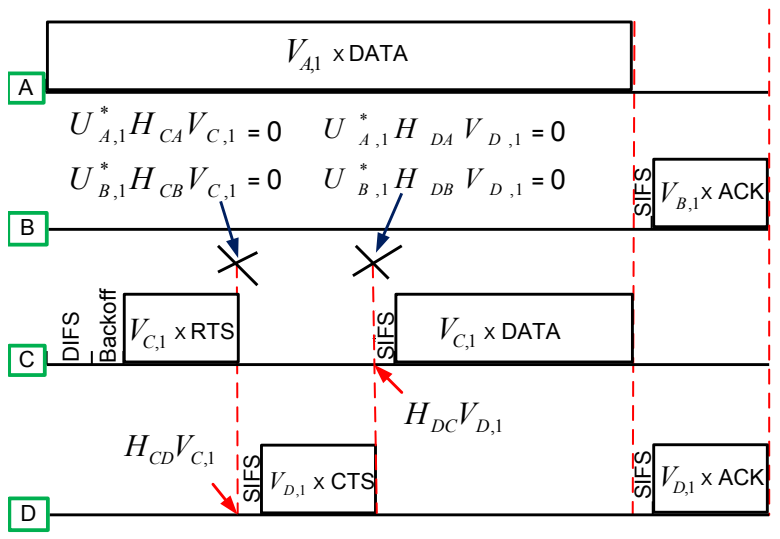

Fig. 4. Four-way handshake to establish parallel communication when the channel is busy.

to concentrate the transmission energy in one directions instead of all directions which improves the received signal strength.

\section{SimUlATION MODEL AND RESUlTS}

Our simulation model is developed and tested under the OPNET Modeler version 17.5-PL5 [14]. The module is available for downloading at [15]. Through three network topologies and different scenarios, we measure the overall network throughput and the average end-to-end delay. We compare the performance of cross-layer framwork with different WLAN amendments (IEEE $802.11 \mathrm{~b} / \mathrm{g} / \mathrm{n}$ ).

\section{A. Implementation of our cross-layer framework in OPNET}

To implement the proposed cross-layer framework we have used the OPNET modeler. For simulation simplification, we have assume that each station in the ad hoc network is equipped with three antennas. The hierarchical structure of any station in the network has: an upper layer modules (transport and application) to generate and receive UDP/CBR traffic, a network module implanting the routing protocol AODV [19], a MAC/cross-layer module, three radio transmitter modules, and three radio receiver modules. Figure 5 illustrates the node model expressed in terms of smaller building blocks called modules. The MAC/cross-layer module contains the CSMA/CA process combined with our cross-layer functioning (see section II). It calculates the transmit and receive filters from the MIMO channel matrix. The radio receiver module implements the Zero-Forcing (ZF) approach including interference cancellation. It estimates and informs the upper layer about the channel matrix. The radio transmit module implements the B-SDM technique ans applies the transmit filter.

\section{B. Simulation parameters}

The parameters used in our simulations are described in Table I. The transmission range is calculated by the following formula:

$\left\{\begin{array}{l}\lambda=0.125 \mathrm{~m} \\ \text { Packet Reception-Power threshold }=-95 \mathrm{dbm}=10^{-12.5} \mathrm{~W} \\ \text { Transmission range }=\sqrt{\frac{5 \times 10^{-3} \times 1 \times(0.125)^{2}}{10^{-12.5} \times 16 \pi^{2}}}=1250.79 \mathrm{~m}\end{array}\right.$ 
TABLE I. Simulation PARAmeters.

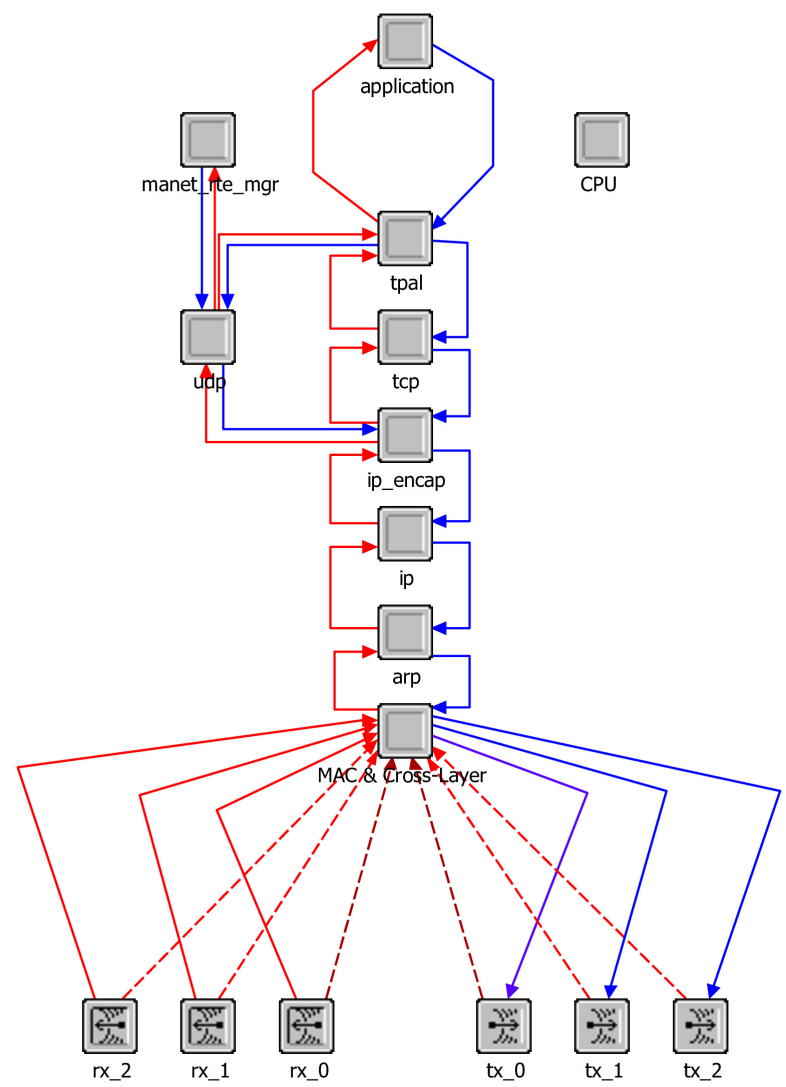

Fig. 5. Node model for MIMOMAX implementation.

\section{Single-hop connection scenario}

In this scenario (Figure 6), we consider a single-hop network connection. Two stations are randomly placed within the transmission range of each other. A station $A$ sends a CBR traffic toward station $B$. We repeat the simulation 100 times, using each time a different seed value. We compare the overall throughput and end-to-end delay of the MIMOMAX framework with IEEE $802.11 \mathrm{~b} / \mathrm{g} / \mathrm{n}$ amendments. For the IEEE 802.11n amendment, we apply the HT-mode with one and two spatial streams (NSS=1 and NSS=2).

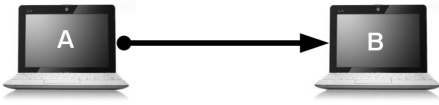

Fig. 6. Single-hop connection.

From Figures 7 and 8, we observe that the HT-mode with NSS $=2$ always performs better than MIMOMAX: up to 2 times more throughput and 2 times less end-to-end delay. This difference can be explained by the low number of DoFs used for the traffic flow transfer. Thus, the HT-mode with NSS $=2$ uses 2 DoFs for data streams transmission while MIMOMAX uses $1 \mathrm{DoF}$ and allocates the remaining DoFs for spatial nulling if concurrent transmissions occur, which leads to underutilization of channel DoFs. We also observe that MIMOMAX

\begin{tabular}{|c|c|c|}
\hline Type & Parameters & Value \\
\hline Radio propagation & $\begin{array}{l}\text { Free Space Model } \\
\text { Wave-Length }(\lambda) \\
\text { Loss Factor } \\
\text { Gain of the transmitter antenna } \\
\text { gain of the receiver antenna }\end{array}$ & $\begin{array}{l}\frac{C}{\text { frequency }} \\
1.0 \\
1.0 \\
1.0\end{array}$ \\
\hline IEEE $802.11 b$ & $\begin{array}{l}\text { number of transmit antennas } \\
\text { Number of receive antennas } \\
\text { physical characteristics } \\
\text { Data Rate } \\
\text { Transmit power } \\
\text { Packet Reception-Power threshold } \\
\text { RTS threshold } \\
\text { Buffer size }\end{array}$ & $\begin{array}{l}1 \\
1 \\
\text { Direct Sequence } \\
11 \mathrm{Mbps} \\
5 \mathrm{~mW} \\
-95 \mathrm{dbm} \\
1500 \text { bytes } \\
1024000 \text { bits }\end{array}$ \\
\hline IEEE $802.11 \mathrm{~g}$ & $\begin{array}{l}\text { number of transmit antennas } \\
\text { Number of receive antennas } \\
\text { physical characteristics } \\
\text { Data Rate } \\
\text { Transmit power } \\
\text { Packet Reception-Power threshold } \\
\text { RTS threshold } \\
\text { Buffer size }\end{array}$ & $\begin{array}{l}1 \\
1 \\
\text { Extended-Rate PHY } \\
48 \mathrm{Mbps} \\
5 \mathrm{~mW} \\
-95 \mathrm{dbm} \\
1500 \text { bytes } \\
1024000 \text { bits } \\
\end{array}$ \\
\hline $\begin{array}{l}\text { IEEE } 802.11 \mathrm{n} \\
\text { and } \\
\text { MIMO Cross-layer }\end{array}$ & $\begin{array}{l}\text { number of transmit antennas } \\
\text { Number of receive antennas } \\
\text { physical characteristics } \\
\text { Data Rate } \\
\text { Transmit power } \\
\text { Packet Reception-Power threshold } \\
\text { RTS threshold } \\
\text { Buffer size } \\
\text { Guard Interval } \\
\text { Greenfield Operation } \\
\text { Number of Spatial Streams (NSS) }\end{array}$ & $\begin{array}{l}3 \\
3 \\
\text { HT PHY } 2.4 G H z \\
216.70 M b p s \\
5 m W \\
-95 d b m \\
1500 b y \text { tes } \\
1024000 \text { bits } \\
400 \text { ns } \\
\text { Enabled } \\
\{1,2\} \\
\end{array}$ \\
\hline Topology & $\begin{array}{l}\text { Area } \\
\text { Number of nodes } \\
\text { Mobility model }\end{array}$ & $\begin{array}{l}10 \mathrm{~km}^{2} \\
\{2,4,30\} \\
\text { Static network }\end{array}$ \\
\hline Routing and Traffic & $\begin{array}{l}\text { Routing Protocol } \\
\text { Type of traffic } \\
\text { Packet size } \\
\text { Packet Inter-arrival time }\end{array}$ & $\begin{array}{l}\text { AODV } \\
\text { CBR/UDP } \\
\text { uniform }(2000,200000) \\
0.002 s\end{array}$ \\
\hline
\end{tabular}

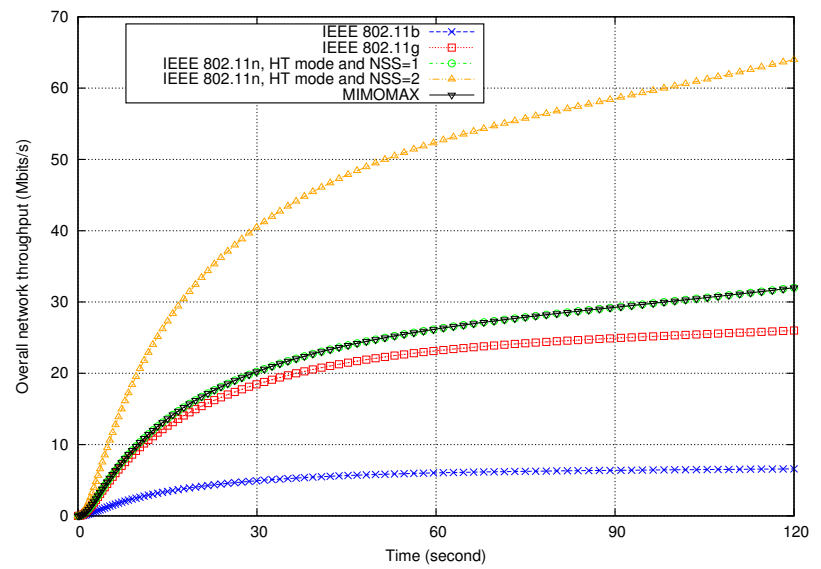

Fig. 7. Throughput of a single-hop wireless network.

and HT-mode with NSS=1 have almost the same performances due to the use of only $1 \mathrm{DoF}$ for data transmission. However, the performance of IEEE 802.11g network is lower than that of HT-mode with NSS=1 and MIMOMAX networks due to the MAC layers enhancements: short guard interval (400ns), frame aggregation, Block ACK and TXOPs. The performance of the IEEE $802.11 \mathrm{~b}$ network is quite poor (the worst performances). 


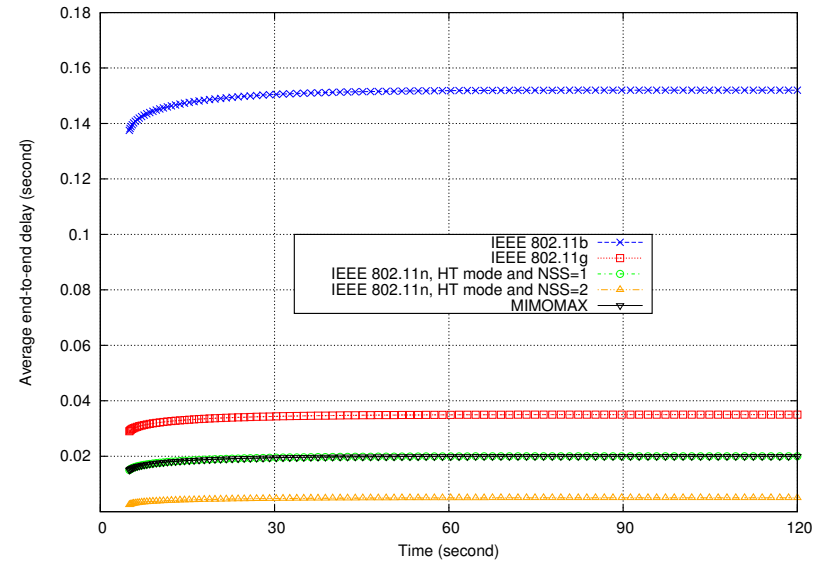

Fig. 8. Delay of a single-hop wireless network.

\section{Cross Transmissions scenario}

In this scenario (Figure 9), we consider two simultaneous transmissions over a single collision domain. Four stations are randomly placed within the transmission range of each other. A station $A$ wants to communicate with $B$ and $C$ with $D$. As in the previous scenario, we repeat the simulation 100 times.

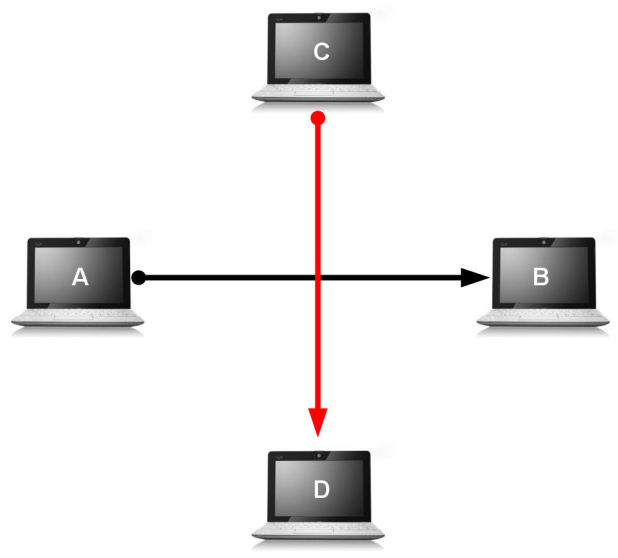

Fig. 9. Concurrent transmissions in the same vicinity area.

From Figures 10 and 11, unlike the previous scenario, we observe that MIMOMAX and HT-mode with NSS $=2$ networks have almost the same overall throughput and end-to-end delay. For the HT-mode with NSS=2 network, each station reserves 2 DoFs for data streams transmission at each slot time in the collision domain. Transmissions $A \longrightarrow B$ and $C \longrightarrow D$ cannot occur simultaneously because there are not enough available DoFs on the channel to admit 4 spatial streams. As discussed in section II, we recall that the channel should have 7 DoFs to allow 4 simultaneous spatial streams transmission. With MIMOMAX, the transmission $A \longrightarrow B$ uses $1 \mathrm{DoF}$ and the other transmission $C \longrightarrow D$ uses 3 DoFs: 1 DoF to transmit and 2 DoFs to signal nulling of (from/to $A$ and $B$ ). To summarize, for both protocols (MIMOMAX and HTmode with NSS=2) and using three antennas per station, the channel supports two spatial streams which explains why their performances are similar. The small difference (about 4\%), can be explained by the fact that with using the HT-mode

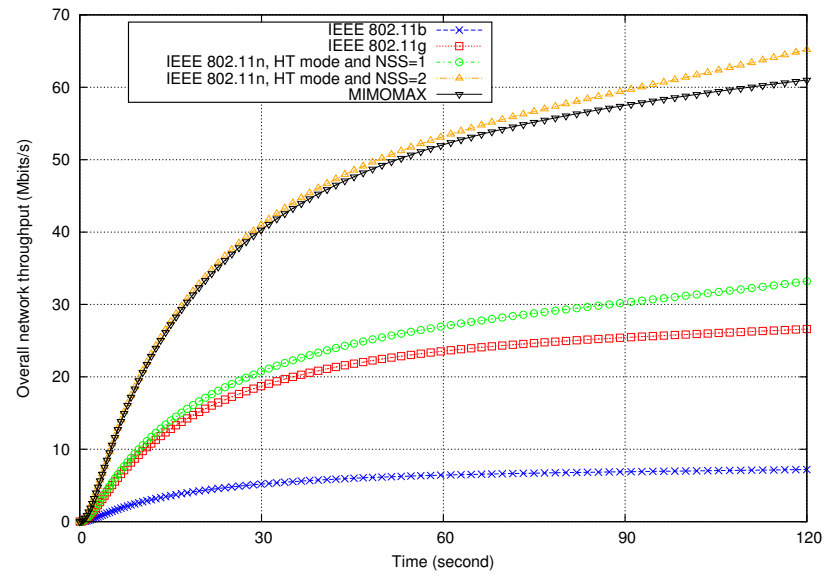

Fig. 10. Throughput of a single-hop wireless network using concurrent transmissions.

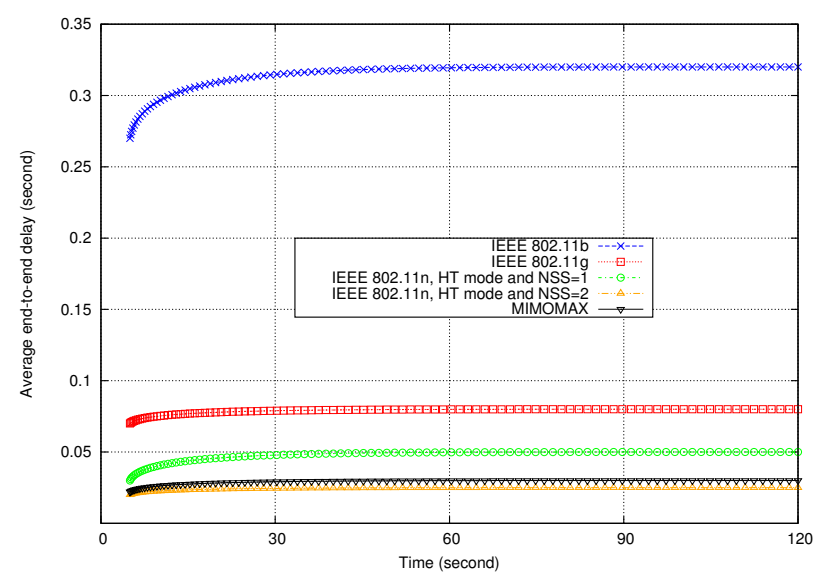

Fig. 11. Delay of a single-hop wireless network using concurrent transmissions.

with NSS=2 there is an overlapping of the backoff decrement periods, which leads to a smaller time interval between two TXOPS.

As the previous scenario, figures 10 and 11 confirm that the two HT-mode with NSS=1 and IEEE 802.11g networks, exhibit almost the same performance with a small difference (about 1-2\%).

\section{E. Multi-hop Network Scenario}

In this scenario we consider a fixed multi-hop network of 30 stations randomly located. We vary the number of CBR/UDP connections form 1 to 10 . We focus on results for 2 and 5 CBR connections. This choice has been motivated by the fact that the network behaves in the same way as with 2 CBR pairs when using 1, 3 and 4 CBR pairs on one hand, and as with 5 CBR pairs (phase-transition) when using 6 up to 10 CBR pairs on the other hand. Each source-destination pair is selected randomly.

Figures 12 and 13 illustrate the total network throughput and the end-to-end delay attainable in the multi-hop network. Unlike the previous scenarios, we observe that MIMOMAX always performs better than all the other protocols. Table II 


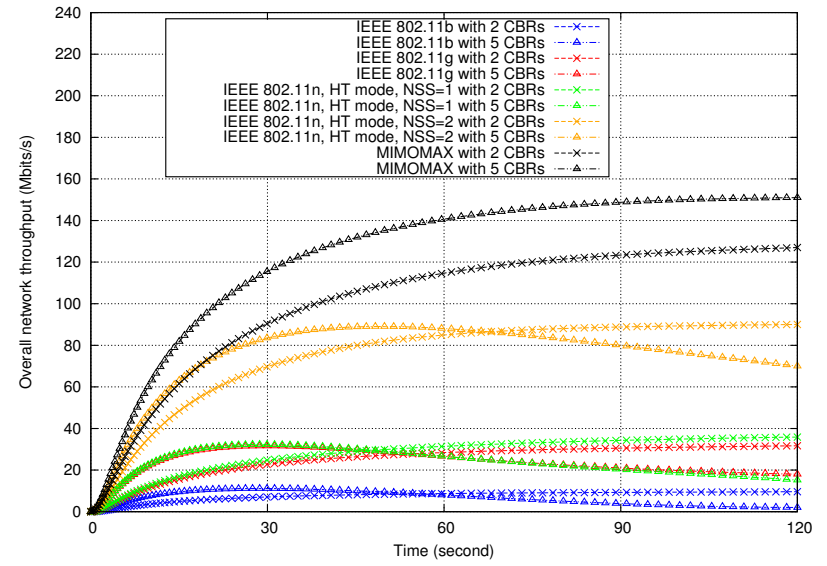

Fig. 12. Throughput of a multi-hop wireless network.

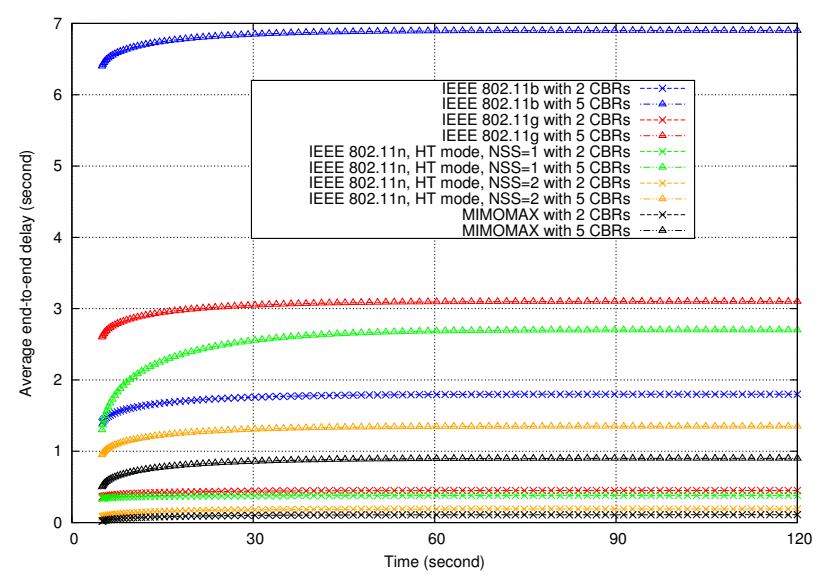

Fig. 13. Delay of a multi-hop wireless network.

TABLE II. OVERALL THROUGHPUT IMPROVEMENT USING THE MIMOMAX.

\begin{tabular}{|l|c|c|}
\hline HT-mode with NSS=2 & 2 CBRs & 5 CBRs \\
HT-mode with NSS=1 & $70 \%$ & $46 \%$ \\
$802.11 \mathrm{~g}$ & $74 \%$ & $87 \%$ \\
$802.11 \mathrm{~b}$ & $88 \%$ & $97 \%$ \\
\hline
\end{tabular}

illustrates the improvements in the overall throughput made by MIMOMAX compared to the other protocols.

The good performance (high throughput and low packet delay) of multi-hop networks based on MIMOMAX can be explained by the traffic/load distribution over multiple nodes in the same collision area with low rates (only one DoF for each data stream transmission). Consequently, as the network load becomes very high, the other protocols drop packets faster than with MIMOMAX. This is mainly due to: 1) the good network traffic distribution with low rate in the case of MIMOMAX, and 2) the high network congestion in the case of other protocols. When the number of the CBR connections increases, the network keeps high performance with MIMOMAX. We can observe severe degradation in throughput and delay of the other protocol after the saturation point is reached. However, MIMOMAX resists to the early network saturation problem which proves its robustness.

\section{CONCLUSION}

MIMO systems have emerged as a promising solution to increase the network capacity and to offer better quality of service for high data rate applications. To fully exploit the benefits of MIMO systems in multi-hop wireless networks, a specially designed MAC protocol is needed. We have proposed a cross-layer design (MIMOMAX) enabling simultaneous transmission on the MIMO channel by multiple stations which are in the same collision domain. In order to evaluate its performance, we have implemented and integrated a new module in OPNET. We have conducted extensive simulation study to provide a comparative analysis of our framework with the IEEE $802.11 \mathrm{~b} / \mathrm{g} / \mathrm{n}$ amendments in single-hop and multihop network context. We have observed that our solution has the best performance in terms of overall network throughput and end-to-end delay under a multi-hop network scenario. It achieves up to $30 \%$ and $87 \%$ as improvements in network throughput compared to the IEEE 802.11n (HT-mode with two spatial streams) and IEEE $802.11 \mathrm{~g}$ standards respectively. When the network load is very high, MIMOMAX remains robust and resists to the early network saturation problem. However, MIMOMAX is less efficient for single-hop networks compared to the other protocols. This is due to the DoFs reservation to null potential concurrent transmissions. As future work, we plan to enhance our solution by using dynamically the available DoFs for high local spatial multiplexing. The variable rate encoding and traffic scheduling issues will be addressed.

\section{REFERENCES}

[1] Part 11: Wireless lan medium access control (mac) and physical layer (phy) specifications. [Online]. Available: http://ieeexplore.ieee.org/xpl/articleDetails.jsp?arnumber=6178212

[2] G. S. Frohn and C. Lindemann, "Analyzing the effective throughput in multi-hop ieee 802.11n networks," Computer Communications, vol. 34, no. 16, pp. 1912-1921, 2011.

[3] T. Ng and S. Liew, "Throughput analysis of ieee802.11 multi-hop ad hoc networks," IEEE/ACM Transactions on Networking, vol. 15, no. 2, pp. 309-322, 2007.

[4] N. Hajlaoui, "On the performance of ieee $802.11 \mathrm{n}$ protocol," in IFIP WMNC, 2012, pp. 64-69.

[5] C. Wang and H. Wei, "Ieee $802.11 \mathrm{n}$ mac enhancement and performance evaluation," Mobile Networks and Applications, vol. 14, no. 6, pp. 477$486,2009$.

[6] K. Sundaresan and R. Sivakumar, "A unified mac layer framework for ad-hoc networks with smart antennas," in MobiHoc, 2004, pp. 244-255.

[7] R. S. K. Sundaresan and M. Ingram, "A fair medium access control protocol for ad-hoc networks with mimo links," in INFOCOM, 2004, pp. 2559-2570.

[8] A. A. A. S. M. J. S. K. B. R. M. Zorzi, J. Zeidler and J. Proakis, "Crosslayer issues in mac protocol design for mimo ad hoc networks," IEEE Wireless Comm. Magazine, special issue on smart antennas, vol. 13, no. 4-13, pp. 62-76, 2006.

[9] J. Park, A. Nandan, M. Gerla, and H. Lee, "Space-mac: Enabling spatial reuse using mimo channel-aware mac," in IEEE Int. Conf. Communications, May 2005, pp. 3642-3646.

[10] T. Tang, M. Park, R. Heath, and S. Nettles, "A joint mimo-ofdm transceiver and mac design for mobile ad hoc networking," in Workshop on Wireless Ad-Hoc Networks, May 2004, pp. 315-319.

[11] C. B. R. Vilzmann and C. Hartmann, "Beammac: A new paradigm for medium access in wireless networks," International Journal of Electronics and Comm., vol. 60, no. 1, pp. 3-7, 2006.

[12] P. R. J. C. Mundarath and B. D. V. Veen, "cross layer scheme for adaptive antenna array based wireless ad hoc networks in multipath environments," Wireless Networks, vol. 13, no. 5, pp. 597-615, 2007. 
[13] M. Zorzi, J. Zeidler, and A. Anderson, "Cross-layer issues in mac protocol design for mimo ad hoc networks," IEEE Trans.Wireless Comm., vol. 13, no. 4, pp. 62-76, 2006.

[14] [Online]. Available: http://www.riverbed.com/productssolutions/products/opnet.html

[15] [Online]. Available: http://www-igm.univ-mlv.fr/ badis/MIMOMAX/

[16] T. Paul and T. Ogunfunmi, "Wireless lan comes of age: Understanding the ieee 802.11n amendment," IEEE Circuits and Systems Magazine, vol. 8, no. 1, pp. 28-54, 2008.

[17] E. L. J. Barry and D. Messerschmitt, Digital Communications. 3rd ed. New York: Springer, 2004.

[18] J. H. et al., "Ldpc coded ofdm with alamouti/svd diversity technique," Wireless Personal Communications, vol. 23, no. 1, p. 183-194, 2002.

[19] E. M. B.-R. C. E. Perkins and S. Das, "Ad hoc ondemand distance vector (aodv) routing," in RFC 3561, 2003. 